\title{
CROMOSOMAS DE CUATRO ESPECIES DE GALACTIA (FABACEAE)
}

\author{
por FLAVIA S. BOSSI y JULIO R. DAVIÑA
}

\begin{abstract}
Summary
The chromosomes of 4 species of Galactia (Fabaceae) have been studied and all of them have $2 n=20$. The chromosome numbers for Galactia marginalis Benth. $(2 n=2 x=20)$, . boavista (Vell.) Burkart $(n=x=10)$ and $G$. benthamiana Micheli $(2 n=2 x=20)$ are presented for the first time. The karyotypes of $G$. striata (Jacq.) Urb. var. striata (20m) and G. benthamiana $(14 m+6 s m)$ are described. Both species can be distinguished by their karyotypic formula.
\end{abstract}

Key words: chromosomes, Fabaceae, Galactia

Palabras clave: cromosomas, Fabaceae, Galactia

\section{Introducción}

El género Galactia P. Browne comprende aproximadamente 50 especies distribuidas en las regiones tropicales y subtropicales de América, Asia y África. En la Argentina crecen alrededor de 14 especies, de las cuales 8 se encuentran en la provincia de Misiones (Burkart, 1971).

Galactia pertenece a la subtribu Diocleinae de la tribu Phaseoleae (Goldblatt, 1981) y sus especies sudamericanas fueron agrupadas por Burkart (1971) en 3 secciones: Galactia, Odonia (Bertol.) Burkart y Collearia (Benth.) Burkart.

Las especies de Galactia son interesantes por presentar cualidades promisorias como forrajeras naturales; entre ellas se destaca Galactia striata (Jacq.) Urb. (Allem \& Valls, 1987), por lo que consideramos de interés la caracterización citogenética de las especies.

Hasta el momento, el género cuenta con pocos recuentos cromosómicos y en la literatura sólo se han encontrado registros para cinco especies argentinas (Goldblatt, 1984, 1985 y 1988 y Goldblatt \& Johnson, 1991). En este trabajo se presentan los resultados de estudios cromosómicos realizados en Galactia marginalis Benth.,

\footnotetext{
${ }^{1}$ Programa de Estudios Florísticos y Genética Vegetal, Fac. Cs. Exact., Qcas. y Nat. U.Na.M. Rivadavia 2370, (3300) Posadas - Misiones, Argentina.
}

G. benthamiana Micheli, G. boavista (Vell.) Burkart y G. striata (Jacq.) Urb. var. striata.

\section{Material y métodos}

Las especies estudiadas fueron coleccionadas en localidades de Misiones y Corrientes, Argentina (Tabla 1). Los ejemplares están depositados en el Herbario de la Universidad Nacional de Misiones (MNES) y en el Herbario del Instituto de Botánica del Nordeste (CTES).

Los estudios mitóticos se realizaron en meristemas radicales, pretratados con 8hidroxiquinoleína 0,002 M, fijados en 5:1 (etanol absoluto : ácido láctico) (Fernández, 1973) y conservados en solución etanol 70 \%. La coloración se realizó según la técnica de Feulgen. Se estudiaron 15 células metafásicas de aproximadamente 10 semillas de cada ejemplar.

El cariotipo se determinó en base al análisis de 10 placas metafásicas y lā nomenclatura utilizada sigue el criterio de Levan et al. (1964). La morfología cromosómica se determinó utilizando el Índice centromérico (Ic) (brazo corto x 100 / longitud total) y los cromosomas se clasificaron en metacéntricos ( $\mathrm{m}$ ) Ic $=50-37,5$ y submetacéntricos $(\mathrm{sm}) I_{\mathrm{c}}=37,5-25$. Para comparar los cariotipos de las distintas especies se utilizaron los Índices de Asimetría de Romero Zarco (1986): $\mathrm{A}_{1}$ (Índice de asimetría intracromosómica) 
$A_{1}=1-\frac{\sum_{i=1}^{n} \frac{b_{i}}{B_{i}}}{n}$, donde $b_{i}=$ brazo corto; $B_{i}=$ brazo largo y $n=$ número de pares cromosómicos y $\mathrm{A}_{2}$ (Índice de asimetría intercromosómica)

$A_{2}=\frac{S}{-}$, siendo $S=$ desvío standard y $X=$ media

de la longitud de todo los cromosomas del set haploide. Ambos índices son independientes del número y tamaño cromosómico. Para establecer las categorías de asimetría de los cariotipos según Stebbins (1971), se consideró la relación entre los cromosomas de máximo y mínimo tamaño, y la proporción de cromosomas con relación de brazos < 2:1, dando un sistema de clasificación consistente en 12 categorías tal como se detalla a continuación.

\begin{tabular}{lllll}
\hline & \multicolumn{4}{c}{$\begin{array}{c}\text { Proporción de cromosomas con } \\
\text { relación de brazos }<2: 1\end{array}$} \\
\cline { 2 - 5 } $\begin{array}{l}\text { Relación tamaño } \\
\text { máximo/mínimo }\end{array}$ & 0,0 & $0,01-0,5$ & $0,51-0,99$ & 1,0 \\
& & & & \\
\hline$<2: 1$ & $1 \mathrm{~A}$ & $2 \mathrm{~A}$ & $3 \mathrm{~A}$ & $4 \mathrm{~A}$ \\
$2: 1-4: 1$ & $1 \mathrm{~B}$ & $2 \mathrm{~B}$ & $3 \mathrm{~B}$ & $4 \mathrm{~B}$ \\
$>4: 1$ & $1 \mathrm{C}$ & $2 \mathrm{C}$ & $3 \mathrm{C}$ & $4 \mathrm{C}$ \\
\hline
\end{tabular}

Las características de los cariotipos fueron comparadas a través de la prueba " $\mathrm{t}$ " para la diferencia de medias.
Para los estudios meióticos se utilizaron botones florales fijados en 5:1 (etanol absoluto: ácido láctico), que se colorearon con orceína acética o carmín acético. El análisis de viabilidad del polen se realizó con carmín glicerina $(1: 1)$.

\section{Resultados}

Los materiales estudiados y los números cromosómicos observados se detallan en la Tabla 1.

Las especies estudiadas mitóticamentes fueron Galactia benthamiana, G. marginalis y $G$. striata. Todas presentaron un número cromosómico de 2n=2x=20 (Fig. 1b, a y c).

Se realizaron estudios meióticos en dos especies: G. striata y G. boavista. G. striata presentó una meiosis regular con 10 bivalentes (Fig. 1e) en metafase I y una viabilidad del polen de 98,32 $\%$. En G. boavista, se analizaron 54 células donde se observó un comportamiento regular de los cromosomas en la meiosis, formando 10 bivalentes (Fig. 1d) y con un promedio de 1,63 de quiasmas por bivalente. Además, el número promedio de bivalentes abiertos por célula fue de 3,71 y la viabilidad del polen fue de $98,25 \%$.

Los datos de los cariotipos de G. striata y G. benthamiana han sido consignados en la tabla 2. El cariotipo de G. striata está constituido por

Tabla 1. Especies analizadas

\begin{tabular}{|c|c|c|c|c|c|}
\hline SECCIÓN & ESPECIES & $2 n$ & $\mathrm{n}$ & Fig. & PROCEDENCIA \\
\hline \multirow[t]{3}{*}{ Odonia } & G. benthamiana Micheli & 20 & & $1 b$ & $\begin{array}{l}\text { Argentina, Misiones, Dpto. Capital, } \\
\text { Posadas. Bossi } 130 \text { (MNES,CTES). } \\
\text { Argentina, Misiones, Dpto. Capital, entre } \\
\text { Fachinal y Ruta 14. Bossi } 179 \text { (MNES). }\end{array}$ \\
\hline & G. marginalis Benth. & 20 & & $1^{\mathrm{a}}$ & $\begin{array}{l}\text { Argentina, Corrientes, Dpto. Capital, Camba } \\
\text { Punta. Daviña } 402 \text { (MNES). }\end{array}$ \\
\hline & $\begin{array}{l}\text { G. striata (Jacq.) Urb. } \\
\text { var. striata }\end{array}$ & 20 & 10 & 1c y e & $\begin{array}{l}\text { Argentina, Misiones, Dpto. Capital, } \\
\text { Nemesio Parma. Bossi } 11 \text { (MNES, CTES). } \\
\text { Argentina, Misiones, Dpto. Candelaria, Campo } \\
\text { San Juan. Daviña } 387 \text { (MNES,CTES). } \\
\text { Argentina, Misiones, Dpto. San Ignacio, Teyú } \\
\text { Cuaré. Bossi } 88 \text { (MNES,CTES). }\end{array}$ \\
\hline Collearia & G. boavista (Vell.) Burkart & & 10 & $1 d$ & $\begin{array}{l}\text { Argentina, Misiones, Dpto. San Ignacio, Teyú } \\
\text { Cuaré. Bossi } 55 \text { (MNES). } \\
\text { Argentina, Misiones, Dpto. San Ignacio, Teyú } \\
\text { Cuaré، Bossi } 90 \text { (MNES,CTES). }\end{array}$ \\
\hline
\end{tabular}


Tabla 2. Caracterización cromosómica de dos especies de Galactia

\begin{tabular}{lcc}
\hline Especie & G. striata & G. benthamiana \\
Cariotipo & $20 \mathrm{~m}$ & $14 \mathrm{~m}+6 \mathrm{sm}$ \\
LTC & $31,42 \mu \mathrm{m}$ & $30,62 \mu \mathrm{m}$ \\
Ic & 44,92 & 41,10 \\
R & $2,10 \mu \mathrm{m}-1,11 \mu \mathrm{m}$ & $2,17 \mu \mathrm{m}-1,08 \mu \mathrm{m}$ \\
$\mathrm{A}_{1}$ & 0,18 & 0,29 \\
$\mathrm{~A}_{2}$ & 0,18 & 0,21 \\
Steb. & $1 \mathrm{~A}$ & $1 \mathrm{~B}$ \\
\hline
\end{tabular}

*LTC: longitud total del complemento; R: rango tamaño cromosómico; $A_{1}$ : índice de asimetría intracromosómica; $\mathrm{A}_{2}$ : índice de asimetría intercromosómica; Steb.: categoría de Stebbins.
20 cromosomas metacéntricos (Fig. 2a) y los cromosomas presentan longitudes entre 1,11 y 2,10 $\mu \mathrm{m}$ (Tabla 3). Por su parte G. benthamiana tiene el cariotipo constituido por $14 \mathrm{~m}+6 \mathrm{sm}$ (Fig. 2b) y las longitudes cromosómicas variaron de 1,08 a 2,17 $\mu \mathrm{m}$ (Tabla 4). En ambos casos se analizaron 10 placas metafásicas.

Con respecto a la simetría, se observó que las especies estudiadas, G. striata $(\mathrm{Ic}=44,92)$ y G. benthamiana $(\mathrm{Ic}=41,10)$ presentaron una diferencia que es significativa según el test de " $t$ " (Tabla 5). El grado de asimetría ( $A_{1}$ ) es mayor en $G$. benthamiana por la variación en las relaciones de brazos (mayor presencia de cromosomas submetacéntricos) con una diferencia altamente significativa (Tabla 5). Tam-

Tabla 3. Medidas cromosómicas expresadas en micras de G. striatı (Bossí, 11)

\begin{tabular}{cccccc}
\hline PAR & $\begin{array}{c}\text { BRAZO CORTO } \\
\pm \text { E.S. }\end{array}$ & $\begin{array}{c}\text { BRAZO LARGO } \\
\pm \text { E.S. }\end{array}$ & $\begin{array}{c}\text { LONGITUD TOTAL } \\
\pm \text { E.S. }\end{array}$ & $\begin{array}{c}\text { ÍNDICE } \\
\text { CENTROMÉRICO }\end{array}$ & TIPO \\
\hline 1 & $0,96-0,18$ & $1,14-0,21$ & $2,10-0,39$ & 45,71 & $\mathrm{~m}$ \\
2 & $0,82-0,19$ & $1,03-0,18$ & $1,85-0,37$ & 44,32 & $\mathrm{~m}$ \\
3 & $0,77-0,14$ & $0,97-0,18$ & $1,74-0,32$ & 44,25 & $\mathrm{~m}$ \\
4 & $0,78-0,14$ & $0,88-0,15$ & $1,66-0,29$ & 46,99 & $\mathrm{~m}$ \\
5 & $0,71-0,11$ & $0,89-0,16$ & $1,60-0,27$ & 44,38 & $\mathrm{~m}$ \\
6 & $0,67-0,11$ & $0,85-0,15$ & $1,52-0,26$ & 44,08 & $\mathrm{~m}$ \\
7 & $0,65-0,13$ & $0,80-0,11$ & $1,45-0,24$ & 44,83 & $\mathrm{~m}$ \\
8 & $0,62-0,14$ & $0,77-0,14$ & $1,39-0,28$ & 44,60 & $\mathrm{~m}$ \\
9 & $0,58-0,13$ & $0,71-0,11$ & $1,29-0,24$ & 44,96 & $\mathrm{~m}$ \\
10 & $0,50-0,11$ & $0,61-0,11$ & $1,11-0,22$ & 45,05 & $\mathrm{~m}$ \\
\hline
\end{tabular}

Tabla 4. Medidas cromosómicas expresadas en micras de G. benthamiana (Bossi, 79)

\begin{tabular}{cccccc}
\hline PAR & $\begin{array}{c}\text { BRAZO CORTO } \\
\pm \text { E.S. }\end{array}$ & $\begin{array}{c}\text { BRAZO LARGO } \\
\pm \text { E.S. }\end{array}$ & $\begin{array}{c}\text { LONGITUD TOTAL } \\
\pm \text { E.S. }\end{array}$ & $\begin{array}{c}\text { ÍNDICE } \\
\text { CENTROMÉRICO }\end{array}$ & TIPO \\
\hline 1 & $0,98-0,17$ & $1,19-0,18$ & $2,17-0,35$ & 45,16 & $\mathrm{~m}$ \\
2 & $0,77-0,09$ & $0,98-0,12$ & $1,75-0,21$ & 44,00 & $\mathrm{~m}$ \\
3 & $0,68-0,06$ & $0,90-0,08$ & $1,58-0,14$ & 43,04 & $\mathrm{~m}$ \\
4 & $0,65-0,07$ & $0,82-0,06$ & $1,47-0,13$ & 44,22 & $\mathrm{~m}$ \\
5 & $0,60-0,06$ & $0,77-0,09$ & $1,37-0,15$ & 43,80 & $\mathrm{~m}$ \\
6 & $0,54-0,09$ & $0,72-0,11$ & $1,26-0,20$ & 42,86 & $\mathrm{~m}$ \\
7 & $0,48-0,09$ & $0,60-0,12$ & $1,08-0,21$ & 44,44 & $\mathrm{~m}$ \\
8 & $0,65-0,10$ & $1,21-0,18$ & $1,86-0,28$ & 34,95 & $\mathrm{sm}$ \\
9 & $0,53-0,07$ & $1,00-0,10$ & $1,53-0,17$ & 34,64 & $\mathrm{sm}$ \\
10 & $0,42-0,05$ & $0,82-0,13$ & $1,24-0,18$ & 33,87 & $\mathrm{sm}$ \\
\hline
\end{tabular}


Tabla 5. Valores de " $\mathrm{t}$ " obtenidos para los parámetros comparados entre G. striata y G. benthamiana

\begin{tabular}{ccl}
\hline Parámetros & Valor de " $\mathrm{t}^{\text {" }}$ obtenido & \multicolumn{1}{c}{ Diferencia } \\
\hline LTC & 0,61 & No significativa \\
Ic & 6,43 & Altamente significativa $(\mathrm{P}=0,01)$ \\
$\mathrm{A}_{1}$ & 5,78 & Altamente significativa $(\mathrm{P}=0,01)$ \\
$\mathrm{A}_{2}$ & 1,46 & No significativa \\
\hline
\end{tabular}

Grados de libertad $=19 ; \mathrm{T}_{0,05}($ tabla $)=2,86 ; \mathrm{T}_{0,01}($ tabla $)=2,09$

*LTC: longitud total del complemento; Ic: Índice centromérico promedio; $\mathrm{A}_{1}$ : índice de asimetría intracromosómica; $\mathrm{A}_{2}$ : índice de asimetría intercromosómica.

bién G. benthamiana presenta una mayor variación en las longitudes cromosómicas $\left(\mathrm{A}_{2}\right)$, aunque este parámetro no presenta una diferencia significativa (Tabla 5).

\section{Discusión}

Se presentan por primera vez los números cromosómicos de 3 especies del género Galactia:

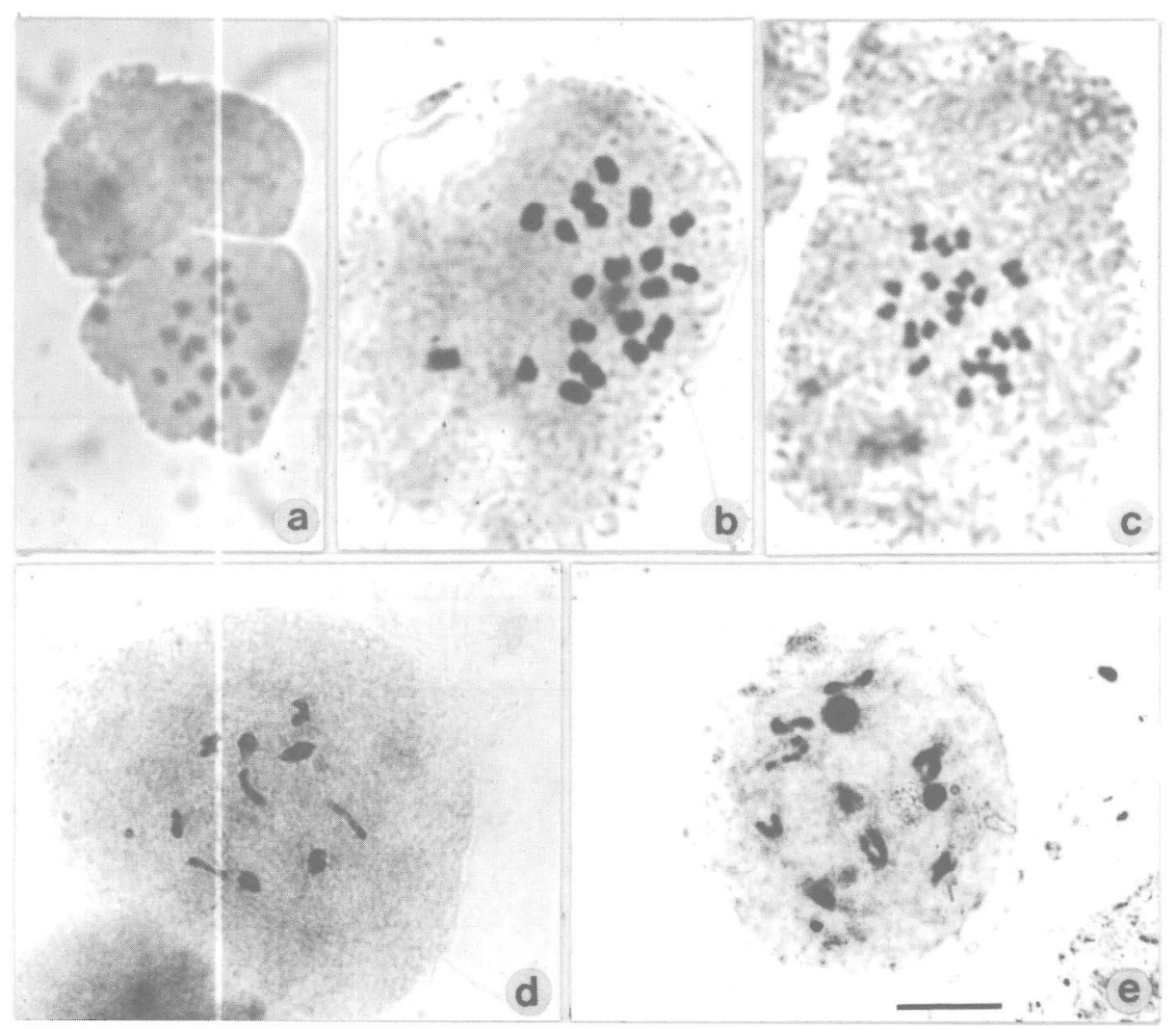

Fig. 1. Galactia marginalis: a, metafase mitótica ( $2 n=20) ; G$. benthamiana: b, metafase mitótica $(2 n=20) ; G$. striata: c, metafase mitótica ( $2 \mathrm{n}=20)$; G. boavista: d, diacinesis con 10 II y G. striata: e, diacinesis con 10 II. La barra representa 5 um. 


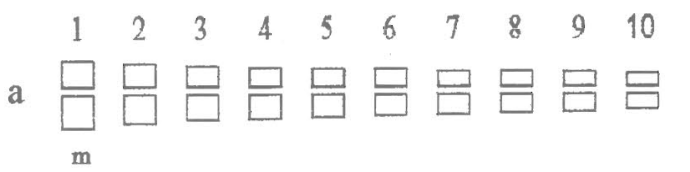

b

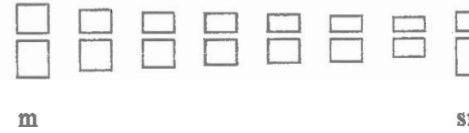

Fig. 2. Idiogramas. a, Galactia striata: 20 m. b, G. benthamiana: $14 \mathrm{~m}+6 \mathrm{sm}$. La barra representa $1 \mu \mathrm{m}$.

G. marginalis posee un área de distribución disyunta (sudoeste de Estados Unidos y México y Sudamérica extratropical: mitad norte de Argentina, Uruguay y sur de Brasil).

G. benthamiana crece desde Brasil central hasta el nordeste y centro de Argentina y G. boavista crece en Brasil central y oriental, Paraguay oriental y el nordeste de Argentina.

G. striata es una especie que ha sido ampliamente estudiada y nuestros resultados en ejemplares de poblaciones argentinas (límite sur de su distribución) confirman los datos obtenidos en estudios previos realizados en poblaciones de Brasil, El Salvador, Honduras y Venezuela (Coleman, 1982; Yeh et al., 1983; Kumar \& Hymowitz, 1989; Torreset al., 1984). Por lo tanto $G$. striata presentaría en toda su área $2 \mathrm{n}=2 \mathrm{x}=20$.

Los cariotipos de las especies analizadas no presentan una diferencia significativa respecto a la longitud total. Las dos especies analizadas difieren en su fórmulas cariotípicas: (20 m) en G. striata y $(14 m+6 \mathrm{sm})$ en $\mathrm{G}$. benthamiana y en ambas especies se distinguen los 2 pares más grandes del complemento. En G. striata, estos 4 cromosomas grandes son metacén tricos y en $G$. benthamiana un par es metacéntrico y el otro submetacéntrico.

Tanto los valores de Ic y de los índices de asimetría de Romero Zarco $\left(\mathrm{A}_{1}\right.$ y $\left.\mathrm{A}_{2}\right)$, las categorías de Stebbins, como las fórmulas cariotípicas indican que los cariotipos son simétricos.

Todas las especies del género estudiadas hasta el momento indican un número cromosómico constante de $2 n=2 x=20$, y por ello el nú- mero básico $x=10$ de Galactia constituye una excepción al $\mathrm{x}=11$ propuesto para la tribu Phaseoleae. Esta reducción se habría establecido tempranamente por disploidía y se habría mantenido constante en todas las especies (Goldblatt, 1981).

De acuerdo con el número básico $\mathrm{x}=10$, las 4 especies estudiadas son diploides y no se registrarían, hasta el momento, poliploides en el género Galactia.

\section{Bibliografía}

ALLEM, A. C. \& J. F. M. VALLS. 1987. Recursos forrageiros nativos do Pantanal mato-grossense. Empresa Brasileira de Pesquisa Agropecuária. Centro Nacional de Recursos Genéticos. Departamento de Difusão de Tecnologia. Brasília, D. F. 339 págs.

BURKART, A. 1971. El género Galactia (Legum.- Phaseoleae) en Sudamérica, con especial referencia a la Argentina y países vecinos. Darwiniana 16 (3-4): 663-796.

COLEMAN, J.R. 1982. Chromosome numbers of Angiosperms collected in the State of São Paulo. Revista Brasil. Genét. 5: 533-549.

FERNÁNDEZ, A. 1973. El ácido láctico como fijador cromosómico. Bol. Soc. Argent. Bot. 15 (2-3): 287-290.

GOLDBLATT, P. 1981. Cytology and the Phylogeny of Leguminosae. En R.M. Polhill \& P.H. Raven eds., Advances in Legume Systematics 2: 427-464. Royal Botanic Gardens, Kew.

1984. Index to plant chromosome numbers 1979-1981. Monogr. Syst. Bot. Missouri Bot. Gard. 8.

1985. Index to plant chromosome numbers 1982-1983. Monogr. Syst. Bot. Missouri Bot. Gard. 13.

1988. Index to plant chromosome numbers 1984-1985. Monogr. Syst. Bot. Missouri Bot. Gard. 23.

\& JOHNSON D.E. 1991. Index to plant chromosome numbers 1988-1989. Monogr. Syst. Bot. Missouri Bot. Gard. 40.

KUMAR,P.S. \& T. HYMOWITZ. 1989. Where are the diploid $(2 \mathrm{n}=2 \mathrm{x}=20)$ genome donors of Glycine Willd. (Leguminosae, Papilionoideae). Euphytica 40: 221-226.

LEVAN, A., K. FREDGA \& A. SANDBERG. 1964. Nomenclature for centromeric position on chromosomes. Hereditas 52: 201-220.

ROMERO ZARCO, C. 1986. A new method for estimating karyotype asymmetry. Taxon 35: 526-530.

STEBBINS, G.L. 1971. Chromosomal evolution in higher plants. Addison-Wesley Publishing Company, 215 págs.

TORRES, Y., G. AGOSTINI \& N. XEN A DE ENRECH. 1984. Revisión taxonómica del género Galactia P. Br. en Venezuela (Leguminosae, Faboideae, Phaseoleae). Acta Biológica Venezuélica 11 (4): 45-93.

YEH, M., F. MAEKAWA \& H. YUASA. 1983. Chromosome numbers of the tribe Phaseoleae, Leguminosae. Res. Inst. Evol. Biol. Sci. Rep. 2: 37-44. 